\title{
THE JAHN-TELLER INSTABILITY WITH ACCIDENTAL DEGENERACY
}

\author{
A. M. STONEHAM* and M. LANNOO†t \\ Theoretical Physics Division, AERE Harwell, Berkshire, United Kingdom
}

(Received 17 October 1968; in revised form 6January 1969)

\begin{abstract}
The Jahn-Teller theorem predicts that orbitally degenerate complexes in solids can lower their energy by a distortion which lowers their symmetry. Exact degeneracy is not necessary for this instability; usually it suffices if the actual separation of the energy levels involved is less than the energy reduction which would result if they were exactly degenerate. We discuss various cases of accidental degeneracy in cubic and tetrahedral systems. With accidental degeneracy it is possible to get mixed distortions which involve both trigonal and tetragonal distortions. These have probably been observed for the negative vacancy in silicon, and possibly for the neutral vacancy in diamond. The theory is applied to both these cases, and it is compared with the earlier qualitative arguments of Watkins.
\end{abstract}

\section{INTRODUCTION}

THE JAHN-TELLER theorem asserts that orbitally degenerate molecules or complexes in solids can lower their energy by a distortion which lowers their symmetry[1]. The instability occurs because the Hamiltonian has matrix elements linear in the distortion, whereas the elastic restoring forces are quadratic in this distortion. The thcorcm is usually applied to cases where the degeneracy of the energy levels is exact and arises from some symmetry of the system. However, exact degeneracy is not necessary for an instability [2]. Usually it is sufficient if the reduction of energy by distortion for exact degeneracy exceeds the actual separation of the levels involved. Near degeneracies can occur even when there is no underlying symmetry reason, and are known as 'accidental' degeneracies.

Accidental degeneracy is particularly interesting because the matrix elements linear in the distortion, responsible for the instability, may connect states which transform according

\footnotetext{
*Present address: Coordinated Science Laboratory, University of Illinois, Urbana, Ill. 61801, U.S.A.

$\dagger$ Permanent address: Institut Supérieur d'Électronique du Nord, Lille, France.

7This article is based on part of a thesis submitted by M.L. for the degree of Doctorat d'Etat en Sciences Physiques (Centre National pour les Recherches Scientifiques No. A.O. 2646).
}

to different representations of the appropriate symmetry group. Usually, with exact degeneracy, matrix elements within one representation alone are considered. For this reason more varied phenomena occur with accidental degeneracy, and inter-level terms are important in the examples considered here.

Opik and Pryce discussed the near degeneracy of the $2 s$ and $2 p$ levels of the $F$ centre. These states are coupled by odd modes parity of $T_{1 u}$ symmetry. For suitable values or parameters the system was unstable with respect to these distortions. The interesting feature here is that a system with full cubic symmetry can lose its inversion symmetry by a Jahn-Teller instability. As the selection rules for optical transitions depend on the parities of the states involved this particular instability may profoundly affect the observed properties of the centre.

This article is mainly concerned with nearly degenerate $E$ and $T$ levels in tetrahedral or cubic symmetry. The calculations show that configurations may be achieved which are of lower symmetry than those obtained ignoring the near degeneracy. Also the inter-level terms may alter the relative importance of trigonal and tetragonal distortions for relatively isolated levels. The results are particularly relevant to defects in the valence crystals diamond, Si and Ge. Two cases are discussed 
in detail. The first is the negative vacancy in Si, where Watkins [3] observed mixed trigonal and tetragonal distortions. His description in terms of a one-electron model is related to a new description, appropriate to a manyelectron model of the type introduced by Coulson and Kearsley[4], for diamond. The second case discussed is the neutral vacancy in diamond. The stress-splitting of the zero phonon line of the GR 1 band shows ano malies, and these may be related to the lowsymmetry distortions discussed here [5].

\section{COMBINED TRIGONAL AND TETRAGONAL DISTORTIONS}

The Jahn-Teller instability arises from matrix elements of the Hamiltonian which are linear in the distortions of the environment. As an example of a system in which the matrix elements of importance connect non-degenerate levels, we discuss in detail the case where a $T_{2}$ level (comprising the states $|\xi>,| \eta>, \mid \zeta>$ ) lies close to an $E$ level (comprising states $|\epsilon\rangle$ and $|\theta\rangle$ ). For zero distortion the $T$ level lies $\Delta$ above the $E$ level. Within these levels the use of symmetry simplifies the matrix elements linear in the distortion to the form:

\begin{tabular}{|c|c|c|c|c|c|c|}
\hline $\begin{array}{l}<\xi \mid \\
<\eta \mid \\
<\zeta \mid\end{array} \mid$ & $\begin{array}{l}G_{E T}(\sqrt{ } 3 \epsilon-\theta) \\
G_{T T} \zeta \\
G_{T T} \eta\end{array}$ & $\begin{aligned} & G_{T T} \zeta \\
- & G_{E T}(\sqrt{ } 3 \epsilon+\theta) \\
& G_{T T} \xi\end{aligned}$ & $\begin{array}{r}G_{T T} \eta \\
G_{T T} \xi \\
2 G_{E T} \theta\end{array}$ & $\begin{array}{l}-\boldsymbol{G}_{T \times} \xi \\
-\boldsymbol{G}_{T \times} \eta \\
2 G_{T X} \zeta\end{array}$ & $\begin{array}{l}+\sqrt{ } 3 G_{T X} \xi \\
-\sqrt{ } 3 G_{T X} \eta\end{array}$ & $(2.1)$ \\
\hline $\begin{array}{r}<\theta \mid \\
<\epsilon \mid\end{array}$ & $\begin{array}{l}-G_{T X} \xi \\
\sqrt{ } 3 G_{T X} \xi\end{array}$ & $\begin{array}{l}-G_{T X} \eta \\
-\sqrt{ } 3 G_{T X} \eta\end{array}$ & $2 G_{T X} \zeta$ & $\begin{array}{l}-G_{E E^{\prime}} \theta \\
G_{E E^{\prime}} \epsilon\end{array}$ & $\begin{array}{l}+G_{E E} \epsilon \\
G_{E E} \theta\end{array}$ & \\
\hline
\end{tabular}

The $G_{I J}$ are coupling coefficients, where $I=$ $E, T$ labels the symmetry of the mode involved, and $J=E, X, T$ indicates the states involved. The matrix elements within the $T$ state have $J=T$ and those connecting the $T$ and $E$ states have $J=X$. The notation $\epsilon, \theta, \xi, \eta, \zeta$ for the normal modes of even parity is standard.*

\footnotetext{
*In tetrahedral symmetry there are, in general, two sets of $T_{2}$ normal modes. We will only discuss vacancy centres with this symmetry; for vacancy centres there is just one set of $T_{2}$ modes, and no ambiguity arises.
}

In addition to these linear terms there are elastic energy terms quadratic in the distortions. The matrix elements of these have the form:

$$
\left[K_{E}\left(\epsilon^{2}+\theta^{2}\right)+K_{T}\left(\xi^{2}+\eta^{2}+\zeta^{2}\right)\right] \hat{1}
$$

where $\hat{1}$ is the unit matrix.

The static distortions of this system will now be discussed, giving the configurations $\mathbf{Q} \equiv(\epsilon, \theta, \xi, \eta, \zeta)$ which minimise the total energy. As general solutions of the secular equation are so complicated as to hide the important features the results will be given for various degrees of intra-level coupling and inter-level coupling. Dynamic effects and spinorbit coupling are ignored.

\subsection{Intra-level coupling only}

We first consider the case when there is no coupling between the $E$ and $T_{2}$ levels, i.e. $G_{7 X}$ is zero. This is the standard case, where there is only coupling within each state. Opik and Pryce concluded that the $E$ states would distort tetragonally, with energy reduction $G_{E E}^{2} / K_{E}$. The $T$ state distorts trigonally if the reduction of energy by trigonal distortion, $G_{T T}^{2} / 3 K_{T}$, exceeds $G_{E T}^{2} / K_{E}$; otherwise a tetra-

gonal distortion results. Mixed tetragonal and trigonal distortions do not provide stable distortions; this result has recently been verified with more general assumptions by Wysling and Müller [6].

\subsection{Inter-level coupling only}

In this section $G_{T X}$ is assumed to dominate, and we treat the case $G_{E E}=G_{E T}=G_{T T}=0$. The eigenvalues can be obtained, as functions of $G_{T X}$. From these configurations of minimum 
energy are found; there are minima for each of the five energy surfaces $E(Q)$. Subsequently Section 2.3 discusses the effects of intra level coupling.

When $G_{E E}, G_{E T}$ and $G_{T T}$ are all zero the eigenvalues for an arbitrary configuration $Q$ are:

$$
\begin{aligned}
E_{a}= & K_{T} q^{2}+\frac{2}{5} \Delta, \\
E_{b, c}= & K_{T} q^{2}-\frac{1}{10} \Delta \\
& +\sqrt{ }\left[\left(\frac{\Delta}{2}\right)^{2}+2 G_{T X}^{2} q^{2}(1 \pm \sqrt{ } 1-\phi)\right] \\
E_{d, e}= & K_{T} q^{2}-\frac{1}{10} \Delta \\
& -\sqrt{ }\left[\left(\frac{\Delta}{2}\right)^{2}+2 G_{T X}^{2} q^{2}(1 \pm \sqrt{ } 1-\phi)\right]
\end{aligned}
$$

in which $q^{2}=\xi^{2}+\eta^{2}+\zeta^{2}$ and $\phi=3\left(\xi^{2} \eta^{2}+\right.$ $\left.\eta^{2} \zeta^{2}+\zeta^{2} \xi^{2}\right) / q^{4}$. The origin of energy is taken to be the centre of gravity of the electronic energy levels for zero distortion. The energy surfaces are labelled $a, b, c, d$ and $e^{*}$.

The results can be conveniently expressed in terms of $F$, a dimensionless parameter defined by:

$$
F=(\Delta / 4) /\left(G_{T X}^{2} / K_{T}\right) .
$$

$F$ is a measure of the inter level coupling, and tends to zero as the coupling becomes large.

It is easily shown that the lowest energy is always found with $|d\rangle$, and is achieved with $\phi=0$. This corresponds to one of six distortions with $(\xi, \eta, \zeta)$ equal to $( \pm q, 0,0)$, $(0, \pm q, 0)$ or $(0,0, \pm q)$. Minimising the lowest eigenvalue, $E_{d}$, with respect to $q$ gives:

$$
\left|q_{0}\right|=\frac{\left|G_{T X}\right|}{K_{T}} \vee\left(1-F^{2}\right)
$$

so there will be an instability in state $|d\rangle$ if

\footnotetext{
${ }^{*} E_{d}$ and $E_{e}$ do not represent separate energy surfaces, but give different sections of two intersecting energy surfaces. $E_{e}$ comprises the upper two branches at the line of intersection and $E_{d}$ the lower two branches. Failure to recognise this can lead one to identify spurious minima, such as the one on the line of intersection $\phi=1$.
}

$|F|<1$. The energy levels for the equilibrium configuration of the ground state $\left(Q=Q_{d 0}\right)$ are given in Fig. 1 as a function of $F$; the

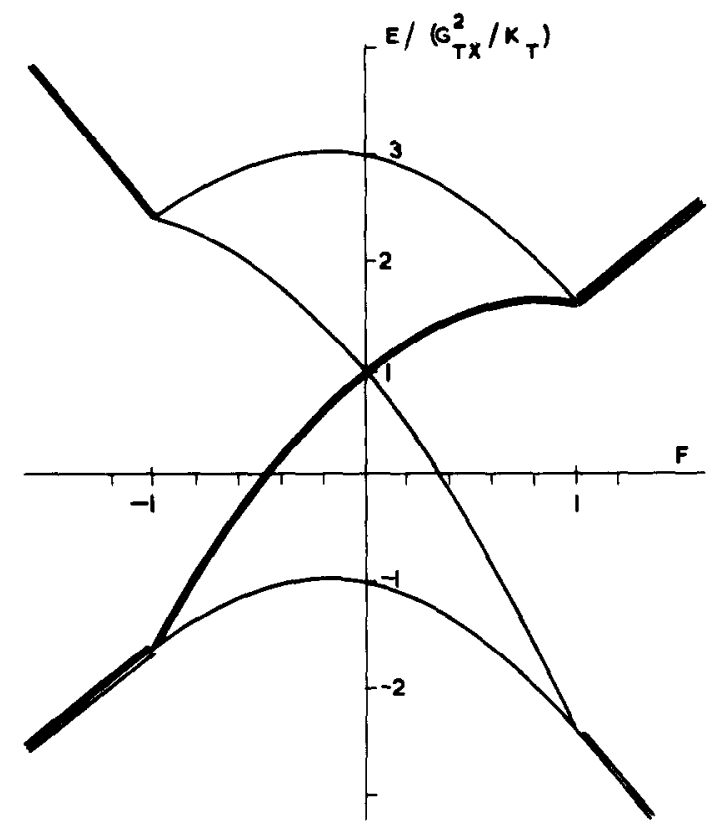

Fig. 1. The energy levels in the $F+T_{2}$ case are shown in units of $G_{T \gamma /}^{2} / K_{T}$ as a function of $F=\Delta /\left(4 G_{T \gamma /}^{2} / K_{r}\right)$. In all cases the levels are calculated for the configuration which minimises the energy in the ground state.

energies are in units of $G_{T X}^{2} / K_{r}$. In this configuration the ground state for the $\left(0,0, q_{0}\right)$ distortion is:

$$
|d\rangle=\sqrt{ }\left(\frac{1}{2}(1+F)\right)|\theta\rangle-\sqrt{ }\left(\frac{1}{2}(1-F)\right)|\zeta\rangle
$$

with energy

$$
E=-\left(G_{T X}^{2} / K_{T}\right)\left(1+\frac{2}{5} F+F^{2}\right) .
$$

The state $|d\rangle$ also shows an extremum with $\phi=\frac{3}{4}$ if $|F|<\frac{3}{4}$. This is a saddle point, not a minimum, and corresponds to a $\left(0, q_{0}^{\prime}, q_{0}^{\prime}\right)$ distortion with energy

$$
-\left(G_{T X}^{2} / K_{T}\right)\left(\frac{3}{4}+\frac{2}{5} F+\frac{4}{3} F^{2}\right) .
$$

Similarly, there is a saddle point in $|e\rangle$ with energy 


$$
-\left(G_{T}^{2} / K_{T}\right)\left(\frac{1}{4}+\frac{2}{5} F+4 F^{2}\right)
$$

if $|F|<(1 / 4)$ and $\phi=3 / 4$.

The other states, $|a\rangle,|b\rangle$ and $|c\rangle$, show no instability from interlevel coupling. They are, of course, still affected by the coupling within the $E$ and $T$ levels.

2.3 Arbitrary intra level and inter level coupling

We follow the method of Öpik and Pryce [2], and express the wave function $\Psi$ as a linear combination of the basis functions, $\psi_{i}$, of the nearly degenerate states:

$$
\begin{aligned}
\Psi & =\sum_{j} a_{j} \psi_{j} \\
\sum a_{j}^{2} & =1 .
\end{aligned}
$$

where $Q$ is one of the normal coordinates $\theta, \epsilon, \xi, \eta$ or $\zeta$. With these equations we can express the $Q$ in terms of the $a_{i}$. Inserting these values for the $Q$ in equation (2.9) we obtain a set of equations in the unknowns $E$ and $a_{i}$. These equations can be solved exactly.

It is easily shown that the extrema obtained with zero inter level coupling always exist. Their energies were given in Section 2.1.

The first mixed solution obtained gives the six possible distortions with $(\theta, \epsilon, \xi, \eta, \zeta)$ equal to $(\rho, 0,0,0, \pm q),\left(-\frac{1}{2} \rho,-\frac{\sqrt{ } 3}{2} \rho, 0, \pm q, 0\right)$ or $\left(-\frac{1}{2} \rho,+\frac{\sqrt{ } 3}{2} \rho, \pm q, 0,0\right)$.

The energy at these minima is:

$$
E=-\frac{G_{T X}^{2}}{K_{T}}\left[\frac{2 F}{5}+\frac{1-\frac{K_{T}}{2 K_{E}}\left\{\frac{G_{E T} \cdot G_{E E}}{G_{T X}^{2}}+\frac{G_{E T}^{2}-\frac{1}{4} G_{E E}^{2}}{G_{T X}^{2}} \cdot F\right\}+F^{2}}{1-\frac{K_{T}}{4 K_{E}}\left(\frac{G_{E T}+\frac{1}{2} G_{E E}}{G_{T X}}\right)^{2}}\right]
$$

and $\rho$ and $q$ are given by:

$$
\begin{aligned}
& \rho=\frac{1}{2}\left[\left(G_{E T}-\frac{1}{2} G_{E E}\right)-F\left(G_{E T}+\frac{1}{2} G_{E E}\right)\right] /\left[\frac{K_{T}}{4}\left(\frac{G_{E T}+\frac{1}{2} G_{E E}}{G_{T X}}\right)^{2}-K_{E}\right] \\
& q=\frac{\left|G_{T X}\right|}{K_{T}} \frac{\sqrt{\left(\left[1-F-\frac{G_{E E}\left(G_{E T}+\frac{1}{2} G_{E E}\right) \cdot K_{T}}{G_{T X}^{2}}\right]\left[1+F-\frac{G_{E E}\left(G_{E T}+\frac{1}{2} G_{E E}\right) \cdot K_{T}}{G_{T X}^{2}}\right]\right.}}{1-\left(\frac{G_{E T}+\frac{1}{2} G_{E E}}{G_{T X}}\right)^{2} \frac{K_{T}}{4 K_{E}}} .
\end{aligned}
$$

The coefficients $a_{j}$ are solutions of the These formulae are only valid if $F$ lies between equations:

$$
E a_{i}=\sum_{j} \mathscr{H}_{i j} a_{j}
$$

where $\mathscr{H}_{i j}$ is the sum of a Jahn-Teller matrix element and an elastic energy term. The extrema of the energy are given by:

$$
0=\sum_{i, j} a_{i} \frac{\partial \mathscr{H}_{i j}}{\partial Q} a_{j}
$$

$$
1-\frac{K_{T}}{4 K_{E}} \frac{G_{E E}\left(G_{E T}+\frac{1}{2} G_{E E}\right)}{G_{T X}^{2}}
$$

and

$$
\frac{K_{T}}{2 K_{E}} \frac{G_{E T}\left(G_{E T}+\frac{1}{2} G_{E E}\right)}{G_{T X}^{2}}-1 ;
$$

these limits exclude any divergences in the denominator of $E$. In the limit of zero intra 
level coupling this state gives the minimum of $|d\rangle$ with $\phi=0$ discussed in Section 2.2. For sufficiently strong interlevel coupling the solution with mixed distortions is the absolute minimum of energy. This result is important. In all cases treated previously the distortions which minimise the energy have been pure trigonal or pure tetragonal; mixed distortions do not provide absolute minima in isolated $E$ or $T$ levels.

Other mixed solutions correspond to $|d\rangle$ and $|e\rangle$ with $\phi=3 / 4$; both are saddle points, and neither of these are minima. The solution for $|d\rangle$ has displacements $(\theta, \epsilon, \xi, \eta, \zeta)$ equal to $\left(-\rho^{\prime}, 0, \pm q^{\prime}, \pm q^{\prime}, \pm p^{\prime}\right)$ and cyclic permutations in the five-coordinate space. The $|e\rangle$ solution has distortions $\left(\boldsymbol{\rho}^{\prime \prime}, 0, \pm q^{\prime \prime}, \pm q^{\prime \prime}, \pm p^{\prime \prime}\right)$ together with cyclic permutations.

\section{OTHER CASES OF NEAR DEGENERACY}

Certain other cases of near degeneracy lead to mixed distortions, and these will be discussed in the present section. Although we shall not discuss all cases of near degeneracy, the ones we omit $(A+A, A+E, E+E)$ are straightforward, and need no special comment. In the $E+E$ case the secular equation can be factorised and, as for an isolated $E$ level, the energies are functions of $\left(\epsilon^{2}+\theta^{2}\right)$ and not of $\epsilon$ or $\theta$ separately. For $A+E$, as has been observed by Elkin and Watkins [7], in an analogous case, the energy does depend on $\epsilon$ and $\theta$ separately; the interaction with the $A$ state effectively produces a cubic anharmonic term which stabilises certain combinations of $\epsilon$ and $\theta$ distortions.

In all our analysis below we refer the final energies to the centroids of the two levels for zero distortion. Our convention is arbitrary, but it simplifies discussion when the order of the levels is inverted.

(a) $A_{1}+T_{2}$ or $A_{2}+T_{1}$

For zero distortion the $T$ level has energy $-\Delta / 4$ and the $A$ level $3 \Delta / 4$. The matrix giving the Jahn-Teller terms is:

$\left[\begin{array}{lcll}G_{E T}(\sqrt{ } 3 \epsilon-\theta) & G_{T T} \zeta & G_{T T} \eta & G_{T X} \xi \\ G_{T T} \zeta & -G_{E T}(\sqrt{3 \epsilon}+\theta) & G_{T T} \xi & G_{T X} \eta \\ G_{T T} \eta & G_{T T} \xi & 2 G_{E T} \theta & G_{T X} \zeta \\ G_{T X} \xi & G_{T X} \eta & G_{T X} \zeta & \end{array}\right]$

There are three types of extremum. The first is the pure tetragonal distortion found for an isolated $T$ level, with energy $-\Delta / 4-G_{E T}^{2} / K_{E}$. There are three equivalent minima of this type. Secondly, there are the four equivalent trigonal distortions with $|\xi|=|\eta|=|\zeta|$. These correspond to the trigonal minima of an isolated $T$ level. Although the distortions which give energy minima for an isolated $T$ level still give extrema, they are only minima when $G_{T X}$ is zero. At the minima in the present case the energy has a complicated form which we give only in the limit $\Delta=0$ :

$$
E=-\left\{\left|G_{T T}\right|+\vee\left(G_{T T}^{2}+3 G_{T X}^{2}\right)\right\}^{2} / 12 K_{T} .
$$

The distortion has, for example:

$$
\xi=\eta=\zeta=\left\{\left|G_{T Y}\right|+\sqrt[V]{ }\left(G_{T T}^{2}+3 G_{T X}^{2}\right)\right\} / 6 K_{T} .
$$

Finally, there is a mixed distortion, with six equivalent minima. One such minimum has $\epsilon=\xi=\eta=0$ and finite values of $\theta$ and $\zeta$ :

$$
\begin{aligned}
& \theta=\frac{G_{E T}}{2 K_{E}} \frac{\left(\Delta+G_{T X}^{2} / K_{T}\right)}{\left(G_{E T}^{2} / K_{E}\right)-\left(G_{T X}^{2} / K_{T}\right)} \\
& \zeta= \pm \frac{G_{T X}}{2 K_{T}} \frac{\left\{\left[\Delta+G_{T X}^{2} / K_{T}\right]\left[G_{T X}^{2} / K_{T}-\Delta-2 G_{E T}^{2} / K_{E}\right]\right\}^{1 / 2}}{\left(G_{E T}^{2} / K_{E}\right)-\left(G_{T X}^{2} / K_{T}\right)}
\end{aligned}
$$


The energy of this mixed state is:

$$
E=\frac{\Delta}{4}-\frac{G_{T X}^{2}}{2 K_{T}}+\frac{1}{4} \frac{\left[\Delta+G_{T X}^{2} / K_{T}\right]\left[2 G_{E X}^{2} / K_{E}-G_{T X}^{2} / K_{T}+\Delta\right]}{G_{E T}^{2} / K_{E}-G_{T X}^{2} / K_{T}} .
$$

This expression is only valid if $\Delta$ lies between $-G_{T X}^{2} / K_{T}$ and $G_{T X}^{2} / K_{T}-2 G_{E T}^{2} / K_{E}$. The last term in $E$ is always finite if this condition is satisfied.

The energies (3.2) and (3.5) are equal for purely interlevel coupling, when $G_{T T}$ and $G_{E T}$ are zero. When there is weak intra level coupling and $\Delta=0$ the trigonal solution has lower energy; in general cases the relative energies depend on the actual magnitudes of the coupling coefficients.

\section{(b) $T_{1}+T_{1}$ or $T_{2}+T_{2}$}

For simplicity we consider the case of interlevel coupling alone. The energy levels are $\pm \Delta / 2$ in the absence of distortion. The relevant part of the Jahn-Teller matrix is:

$$
\left[\begin{array}{ccc}
G_{E X}(\sqrt{ } 3 \epsilon-\theta) & G_{T X} \zeta & G_{T X} \eta \\
G_{T X} \zeta & -G_{E X}(\sqrt{ } 3 \epsilon+\theta) & G_{T X} \xi \\
G_{T X} \eta & G_{T X} \xi & 2 G_{E X} \theta
\end{array}\right] .
$$

The solutions are analogous to those for an isolated $T$ level, in that there are both tetragonal and trigonal extrema, and the mixed distortions have energies intermediate between these values. Further, the trigonal distortion is again favoured if $G_{T X}^{2} / 3 K_{T}$ exceeds $G_{E X}^{2} / K_{E}$. However, there are now six equivalent tetragonal minima, with energy

$$
E=-\frac{G_{E: X}^{2}}{K_{E}}\left\{1+\left(\frac{\Delta / 4}{G_{E X}^{2} / K_{E}}\right)^{2}\right\}
$$

provided $|\Delta|<4 G_{E X}^{2} / K_{E}$. The distortions here are, for instance:

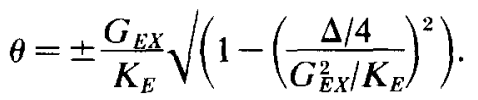

When $|\Delta|<4 G_{T X}^{2} / 3 K_{T}$ there are eight equivalent trigonal minima, with energy

$$
E=-\frac{G_{T X}^{2}}{3 K_{T}}\left\{1+\left(\frac{\Delta / 4}{G_{T X}^{2} / 3 K_{T}}\right)^{2}\right\}
$$

and with distortions such as:

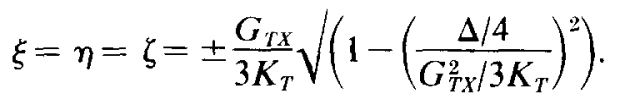

The degeneracies which are associated with the different possible signs of $\theta$ and of $\xi, \eta$ and $\zeta$ may be lifted by anharmonic terms or by the intralevel coupling. Inclusion of these extra terms greatly increases the difficulty of the problem, and we have not examined these features in detail. When there is both interlevel and intralevel coupling, mixed distortions can occur. This can be easily seen by considering the effect of weak coupling to $E$ modes from intralevel coupling when interlevel coupling gives a trigonal distortion. However, the general case is extremely complicated, and we have not treated the most general form.

\section{(c) $T_{1}+T_{2}$}

Again we consider zero intralevel coupling. When the distortions are zero the $T_{2}$ level lies $\Delta$ above the $T_{1}$ level. The interlevel part of the matrix linear in the distortions is:

$\left[\begin{array}{ccc}-G_{E X}(\sqrt{ } 3 \theta+\epsilon) & -G_{T X} \zeta & G_{T X} \eta \\ G_{T X} \zeta & +G_{E X}(\sqrt{ } 3 \theta-\epsilon) & -G_{T X} \xi \\ -G_{T X} \eta & G_{T X} \xi & 2 G_{E X} \epsilon\end{array}\right]$

The results are completely different from the $\left(T_{1}+T_{1}\right)$ and $\left(T_{2}+T_{2}\right)$ cases. There are three classes of distortion which give minima 
in $E(\mathbf{Q})$. As the solutions are complicated, we introduce the abbreviations:

$$
\begin{aligned}
& \gamma_{E}=G_{E X}^{2} / K_{E} \\
& \gamma_{T}=G_{T X}^{2} / K_{T} \\
& \gamma_{2}=\left(3 \gamma_{E}+\gamma_{T}\right) / 4 \\
& \gamma_{3}=\gamma_{T}\left(\gamma_{T}+2 \gamma_{E}\right) /\left(4 \gamma_{T}-\gamma_{E}\right)
\end{aligned}
$$

The first solution gives six equivalent minima. These are orthorhombic, one of them having $\epsilon$ finite and $\theta=\xi=\eta=\zeta=0$. Its energy is

$$
E_{1}=-\gamma_{E}\left\{1+\left(\frac{\Delta}{4 \gamma_{E}}\right)^{2}\right\}
$$

and the distortion is:

$$
\epsilon= \pm \frac{G_{E X}}{K_{E}} \sqrt{\left(1-\left(\frac{\Delta}{4 \gamma_{E}}\right)^{2}\right)}
$$

These are valid when $4 \gamma_{E^{\prime}}>|\Delta|$. The second solution gives a mixed distortion, with $\theta$ and $\zeta$ finite, and $\epsilon=\xi=\eta=0$. It has $C_{2}$ symmetry, and energy:

$$
E_{2}=-\gamma_{2}\left\{1+\left(\frac{\Delta}{4 \gamma_{2}}\right)^{2}\right\}
$$

valid when $4 \gamma_{2}>|\Delta|$. There are 12 such distortions, for example:

$$
\left.\begin{array}{l}
\theta= \pm \frac{\sqrt{ } 3}{2} \frac{G_{E X}}{K_{E}} \sqrt{\left(1-\left(\frac{\Delta}{4 \gamma_{2}}\right)^{2}\right)} \\
\zeta= \pm \frac{1}{2} \frac{G_{T X}}{K_{T}} \sqrt{\left(1-\left(\frac{\Delta}{4 \gamma_{2}}\right)^{2}\right)}
\end{array}\right\} .
$$

The third solution also gives a mixed distortion, but with $\theta=\xi=0$, and $|\xi|=|\eta|$. The energy at this minimum is:

$$
E_{3}=-\gamma_{3}\left\{1+\left(\frac{\Delta}{4 \gamma_{3}}\right)^{2}\right\}
$$

valid when $4 \gamma_{3}>|\Delta|$ and $4 \gamma_{T}>\gamma_{E}$. In this case the symmetry is $C_{1}$, and there are 24 equivalent sets of distortions, corresponding to the various permutations of signs and interchange of cube axes with:

$$
\left.\begin{array}{l}
|\epsilon|=3 \frac{G_{E X}}{K_{E}} \sqrt{\left(1-\left(\frac{\Delta}{4 \gamma_{3}}\right)^{2}\right) \frac{\gamma_{T}}{4 \gamma_{T}-\gamma_{E}}} \\
|\xi|=|\eta| \\
=\frac{G_{T X}}{K_{T}}\left[1-\frac{\Delta}{4 \gamma_{3}}\right] \frac{V\left(\left(2 \gamma_{T}+\gamma_{E}\right)\left(\gamma_{T}-\gamma_{E}\right)\right)}{4 \gamma_{T}-\gamma_{E}}
\end{array}\right\}
$$

To summarise, we have:

$$
\begin{array}{ll}
\text { (i) } \gamma_{E}<\gamma_{T} & : E_{2}<E_{3}<E_{1} \\
\text { (ii) } \frac{1}{4} \gamma_{E}<\gamma_{T}<\gamma_{E} & : E_{3}<E_{1}<E_{2} \\
\text { (iii) } \gamma_{T}<\frac{1}{4} \gamma_{E} & : E_{1}<E_{2} .
\end{array}
$$

(d) $E+T_{1}$

This is very similar to the example given in detail in Section 2, $\left(E+T_{2}\right)$. The Jahn-Teller matrix elements differ from the earlier example in that the elements involving $|\epsilon\rangle$ and $|\theta\rangle$ should be interchanged, and those in $|\theta\rangle$ changed in sign. The energy reductions and distortions are not affected, but the wave functions are altered. The results for $T_{1}+E$ can be obtained from the earlier results by the substitution of $-|\theta\rangle$ for $|\epsilon\rangle$ and of $|\epsilon\rangle$ for $|\theta\rangle$.

\section{COMPARISON WITH EXPERIMENT}

In this section we discuss briefly a number of systems which illustrate the Jahn-Teller effect for near, rather than exact, degeneracy. The systems described all show mixed trigonal and tetragonal distortions. Examples are the negative vacancy in silicon [3] and the transition ions $\mathrm{Pd}^{-}$and $\mathrm{Pt}^{-}$in silicon and $\mathrm{Ni}^{-}$in germanium [8]. The neutral vacancy in diamond may exhibit mixed distortions also, but the evidence is less direct. We will only discuss the vacancy centres in valence crystals, and will outline the reasons for expecting them to show the effects of near degeneracy. Watkins has emphasised [9, for example] that the Jahn-Teller terms are particularly large in silicon.

It is easy to show that, for these vacancy centres, there are likely to be energy levels nearly degenerate with the ground state of the centre. The electronic properties of vacancies in valence crystals are largely 
determined by the so-called 'defect electrons'. In the neutral vacancy, for instance, four bonds were broken in forming the centre; the four electrons on the neighbours which previously participated in the bonds are the defect electrons. New molecular orbitals can be formed by taking linear combinations of the broken bond orbitals. In particular the molecular orbital of lowest energy, $|v\rangle$, has $A_{1}$ symmetry; above it are three degenerate $T_{2}$ orbitals, $\left|t_{x}\right\rangle,\left|t_{y}\right\rangle$ and $\left|t_{z}\right\rangle$. These oneelectron orbitals are filled successively by the defect electrons, giving electronic configurations $v^{n t} t^{m}$. However, from any one configuration a number of many-electron states may be constructed, and these states will, in general be close in energy [4]. For the negative vacancy in silicon three states of the same spin $\left({ }^{2} E,{ }^{2} T_{1}\right.$ and $\left.{ }^{2} T_{2}\right)$ and one other $\left({ }^{4} A_{2}\right)$ derive from the configuration $v^{2} t^{3}$. For the neutral vacancy in diamond there are again three states of the same multiplicity $\left({ }^{1} E,{ }^{1} T_{2}\right.$ and ${ }^{\prime} A_{1}$ ) and one of different multiplicity (" $\left.T_{1}\right)$ which all derive from $v^{2} t^{2}$.

In the simplest models of the vacancy centres all states which derive from the same configuration $v^{t \prime} t^{\prime \prime}$ are exactly degenerate. The degeneracy will be removed by the electron-electron interaction, which admixes states of the same symmetry derived from $v^{n-1} t^{m+1}, \quad v^{n-2} t^{m+2}, \ldots$. Such configuration interaction is very important in diamond and, as we shall show, may cause large enough splittings to inhibit Jahn-Teller instabilities. On the other hand, in silicon, Watkins has found that the configuration interaction is much smaller, and may often be ignored. For this reason he has been able to interpret his data in terms of a one-electron model, rather than in terms of Jahn-Teller coupling between many-electron states which are close in energy. The two pictures have much in common, for example, both recognise that the matrix elements which cause the instability are linear in the distortions, and indeed the one-electron model can be considered as a special case of the many-electron picture.
We shall not compare the two viewpoints in detail here, but merely observe that Watkins' model is particularly useful for understanding the nature of the ground states of these centres a posteriori, when the configuration interaction is known to be weak. The approach via the many-electron states of low energy is more appropriate when one wants to predict the ground state a priori, since this approach involves a more general formulation of the Jahn-Teller problem and also detailed consideration of the energy separations of the various many electron states.

We conclude this section by discussing the neutral vacancy in diamond, since the centre has been studied extensively theoretically, and it is possible to give some idea of the relative importance of the configuration interaction and Jahn-Teller terms. Although the Jahn-Teller terms dominate in silicon, the situation is less clear in diamond. The evidence for mixed trigonal and tetragonal distortions of the neutral vacancy in diamond comes from the stress splitting of the zerophonon line of the GR1 band, often attributed to the neutral vacancy $[4,5]$. Theoretical treatments suggest that the ground state is ${ }^{1} E$, separated by some $0.05 \mathrm{eV}$ from the ${ }^{3} T_{1}$ state; the next state, ${ }^{1} T_{2}$, is about $2 \mathrm{eV}$ higher in energy. The GR1 band is attributed to transitions between the ${ }^{1} E$ and ${ }^{1} T_{2}$ states. The interlevel coupling which gives rise to the mixed distortions can occur in two obvious ways: coupling between ${ }^{1} E$ and ${ }^{3} T_{1}$, or coupling between ${ }^{1} E$ and ${ }^{1} T_{2}$. Although ${ }^{1} E$ and ${ }^{3} T_{1}$ are very close in energy, their coupling depends on the spin-orbit coupling. In diamond the spin-orbit coupling is very weak, and the difference in multiplicity effectively reduces $G_{T X}$ by about $10^{-3}$. The interaction is then too weak; mixed distortions would only occur if the two levels were only separated by about $10^{-7} \mathrm{eV}$. The ${ }^{1} E$ and ${ }^{1} T_{2}$ levels have a larger separation, and should exhibit an instability if $G_{T X}$ exceeds about $4 \mathrm{eV} / \AA$. Calculations based on the point-ion model $[10,11]$ give $2 \cdot 6 \mathrm{eV} / \AA$, and those based on the 
LCAO method[12] give $0 \cdot 26 \mathrm{eV} / \AA$. These results are not conclusive; what the calculation does emphasise is that, if the interpretation of the experiments is correct, the JahnTeller coupling between the ${ }^{1} E$ and ${ }^{1} T_{2}$ states is only just large enough to cause the instability.

To summarise, we see that near degeneracies occur quite naturally for vacancy centres in valence crystals. The degeneracy of states derived from any one configuration is removed by the interaction between the defect electrons. In silicon the Jahn-Teller terms are much larger than the splittings due to the electron-electron interaction, but the two terms are comparable for diamond. The near degeneracies which are important in both these cases involve states with the same spin. It is clear, however, that in systems where there is appreciable spin-orbit coupling the near degeneracy of states with different multiplicity may be important.

\section{CONCLUSION}

Nearly degenerate levels are coupled by terms linear in the distortions of the environment. When the coupling is sufficiently strong, and the levels are sufficiently close, a JahnTeller instability occurs. In combination with this the Jahn-Teller coupling within the levels can lead to mixed trigonal and tetragonal distortions; these cannot occur if there is no coupling between the levels.
These mixed distortions are of importance for defects in valence crystals, and offer an explanation of the spin resonance data for the negative vacancy in Si. It is possible that the low symmetry distortions also explain the unusual stress-splitting of the zero phonon line of the GR 1 line in diamond.

Acknowledgements-We are indebted to Dr. G. D. Watkins for a stimulating correspondence, and to Dr. K. A. Müller for a copy of his work prior to publication.

\section{REFERENCES}

1. JAHN H. and TELLER E., Proc. R. Soc, A161, 220 (1937).

2. OPIK U. and PRYCE M. H. L., Proc. R. Soc. A238, 425 (1957).

3. WATKINS G. D., Radiation Damage in Semiconductors, pp. 97-113. Dunod, Paris (1964).

4. COULSON C. A. and KEARSLEY M. J., Proc. $R$. Soc. A241, 433 (1957).

5. LANNOO M. and STONEHAM A. M., J. Phys. Chem. Solids 29, 1987 (1968).

6. WYSLING P. and MÜLLER K. A., Phys. Rev. 173, 327 (1968).

7. ELKIN E. L. and WATKINS G. D., Phys. Rev. 174, 881 (1968).

8. LUDWIG G. W. and WOODBURY H., Solid St. Phys. 13, 223 (1962).

9. LIDIARD, A. B., Report of Panel Discussion in Radiation Damage in Semiconductors, p. 335. Dunod, Paris (1964).

10. STONEHAM A. M., Proc. Phys. Soc. 88, 135 (1966).

11. LIDIARD A. B. and STONEHAM A. M., Science Techn. Industrial Diamonds 1, 1 (1966).

12. FRIEDEL J., LANNOO $M$. and LFMAN G., Phys. Rev. 164, 1056 (1967). 